\title{
Treatment of pancreatic cancer with epidermal growth factor receptor-targeted therapy
}

\author{
This article was published in the following Dove Press journal: \\ Biologics:Targets \& Therapy \\ 3 September 2009 \\ Number of times this article has been viewed
}

Bryan A Faller

Barbara Burtness

Department of Medical Oncology, Fox Chase Cancer Center,

Philadelphia, PA, USA
Correspondence: Barbara Burtness Department of Medical Oncology, Division of Medical Sciences, 333 Cottman Avenue, Fox Chase Cancer Center, Philadelphia, PA I91।I, USA

$\mathrm{Tel}+\mathrm{I}(2 \mathrm{I}$ ) 728-3023

$\mathrm{Fax}+\mathrm{I}(215)$ 728-3639

Email barbara.burtness@fccc.edu

\begin{abstract}
Pancreatic adenocarcinoma is a common malignancy that remains refractory to available therapies. Gemcitabine has long been the standard, first-line agent in advanced disease. The epidermal growth factor receptor (EGFR) is a commonly expressed target in pancreatic cancer that is involved in tumor proliferation, metastasis, and induction of angiogenesis. The addition of the EGFR inhibitor erlotinib to gemcitabine has recently been demonstrated to provide a small, yet statistically significant, survival benefit in advanced disease. This has prompted further research into the applications of EGFR-targeted therapy in pancreatic cancer, albeit with disappointing results. Resistance to these therapies seems highly prevalent and has been implicated in their limited efficacy. The development of rash is associated with treatment efficacy and suggests that predictive factors may one day be identified to guide appropriate patient selection for these agents. Preclinical research has shown promise that resistance to EGFR-targeted therapies can be overcome through a variety of approaches. Application of this research in clinical trials may ultimately yield an unquestioned role for EGFR-targeted therapy in the management of this disease.
\end{abstract}

Keywords: cetuximab, drug resistance, epidermal growth factor receptor, erlotinib, gemcitabine, pancreatic cancer

\section{Introduction}

Adenocarcinoma of the pancreas remains a highly treatment-refractory malignancy. Although it is only the thirteenth most common cause of cancer worldwide, it is the eighth most common cause of cancer-related death. ${ }^{1}$ Gemcitabine remains the standard first-line cytotoxic agent for metastatic disease since demonstrating superiority over fluorouracil monotherapy. ${ }^{2}$ Median survival of gemcitabine-treated patients with advanced disease is 5 to 6 months. Recently, a small, statistically significant improvement in overall survival (OS) was demonstrated by the addition of EGFRtargeted therapy to gemcitabine, bringing to question a new standard regimen. ${ }^{3}$ This finding has prompted continued exploration of the role of EGFR-targeted therapies in the management of pancreatic adenocarcinoma. The modest nature of the improvement resulting from erlotinib therapy, however, has also prompted efforts to understand the mechanisms of resistance to EGFR inhibition, which seem to be highly prevalent in this disease.

\section{EGFR biology in pancreatic adenocarcinoma}

The EGFR, also known as ErbB1, is a $170 \mathrm{kDa}$ protein belonging to the four-member ErbB family of transmembrane tyrosine kinase growth factor receptors. Binding by 
a number of ligands induces conformational alterations in the single chain receptor, allowing dimerization or oligomerization with other EGFR molecules or other members of the ErbB family. Multimer formation leads to asymmetric autophosphorylation of a tyrosine residue on the intracellular portion of one of the molecules, allowing the receptor to recruit docking proteins and signal transduction molecules. The resulting cascades, involving the Ras/mitogen-activating protein (MAP) kinase pathway, the Phosphoinositide-3 kinase (PI3K)/Akt pathway, Src family kinases, and signal transducers and activator of transcription (STAT) proteins, result in upregulation of mitogenic, antiapoptotic, angiogenic, and pro-invasive cellular mechanisms. ${ }^{4}$ Several routes to aberrant receptor activity in malignancy have been described, including receptor over-expression, mutation, liganddependent receptor dimerization, and ligand-independent activation. As an example, activating mutations in the gene for the downstream regulating protein, $K R A S$, have been found to be highly prevalent in pancreatic cancer. ${ }^{5,6}$ Evidence from genetically engineered mouse models supports the role of these activating $K R A S$ mutations in the pathogenesis of pancreatic cancer. $^{7}$

EGFR is expressed in $30 \%$ to $89 \%$ of pancreatic cancers assayed by immunohistochemistry techniques. ${ }^{8}{ }^{89}$ Its expression had been shown to correlate with worse outcome and more aggressive disease in small case series. ${ }^{10} \mathrm{~A}$ more recent review of pancreatic cancer cases at the Ohio State University published in 2006, however, found conflicting data. In this series, EGFR was expressed in $69 \%$ of the 71 cases. Its expression did not correlate with tumor grade, size, lymphatic involvement, or survival. In fact, there was a nonstatistically significant trend for longer survival in the patients whose tumors expressed EGFR (median OS: 15.2 months vs 8.3 months). ${ }^{11}$

Exposure of pancreatic cancer cell lines to gemcitabine results in increased phosphorylation and thus activition of EGFR. ${ }^{12}$ This can be effectively blocked by tyrosine kinase inhibitors of EGFR, leading to tumor apoptosis. ${ }^{13,14}$ In xenograft models of pancreatic cancer, the combination of gemcitabine and EGFR-targeted therapy significantly inhibits lymph node and liver metastasis and results in improved overall survival. ${ }^{13}$

A major partner of EGFR, HER-2 (ErbB2), has also been shown to be overexpressed in numerous human cancers and is associated with multiple drug resistance, higher metastatic potential, and decreased patient survival. ${ }^{15}$ Aberrant HER-2 expression in pancreatic cancer has been reported in a number of studies, with a prevalence ranging from $7 \%$ to $58 \% .{ }^{15}$ Expression of EGFR-related protein (ERRP), an effective pan-erbB inhibitor, has been found to correlate inversely with the degree of differentiation in pancreatic cancer. Likewise, low levels of ERRP are associated with poor clinical outcome. ${ }^{16}$

\section{EGFR inhibitors}

Small molecular inhibitors of EGFR, such as erlotinib and gefitinib, act by competing with adenosine- 5 -triphosphate (ATP) for the intracellular tyrosine kinase domain of the receptor. They thereby inhibit EGFR autophosphorylation, and thus downstream signaling. ${ }^{17}$ These agents are administered orally and have dose-limiting toxicities of a characteristic rash, diarrhea, and an interstitial lung diseaselike syndrome, possibly below the most effective dose for some agents. Significant pharmacogenomic variability in absorption and metabolism has been described. ${ }^{18}$ Several small-molecule EGFR tyrosine kinase inhibitors (TKI) can block multiple growth factor receptor tyrosine kinases, including other members of the ErbB family, or the vascular endothelial growth factor receptor (VEGFR). Lapatinib is one such molecule that reversibly inhibits both Her2 (ErbB2) and, less potently, EGFR. Small-molecule TKIs have the theoretical advantage of inhibiting ligand-independent activity of EGFR. Erlotinib and gefitinib have been associated with greater efficacy in nonsmall cell lung cancers (NSCLC) bearing activating mutations in the EGFR tyrosine kinase domain. ${ }^{19}$

Anti-EGFR monoclonal antibodies have been developed that competitively bind the extracellular ligand-binding region of the receptor in its inactivated position. Once bound, they prevent ligand binding and receptor dimerization. These agents therefore block endogenous ligand activation of EGFR in a highly specific manner, but may fail to inhibit ligand-independent activity. Two such examples include cetuximab, a human-murine IgG1 chimeric antibody, and panitumumab, a fully humanized IgG2 antibody. Both agents are well tolerated with predominant toxicities including rash, diarrhea, and hypomagnesemia. Serious hypersensitivity reactions, associated with pre-existing immunoglobulin (Ig)E antibodies against galactose-alpha-1,3-galactose, may occur during infusion of cetuximab; ${ }^{20}$ hypersensitivity reactions occur far less commonly with panitumumab, and the mechanism of these is not identified. ${ }^{21}$

\section{EGFR tyrosine kinase inhibitors in advanced pancreatic cancer}

The National Cancer Institute of Canada Clinical Trials Group (NCIC CTG) performed the only trial to demonstrate 
OS benefit from the addition of EGFR targeted therapy to standard cytotoxic gemcitabine therapy in pancreatic cancer. ${ }^{3}$ It was a randomized, placebo-controlled, phase III trial comparing erlotinib with gemcitabine to gemcitabine alone in patients with locally advanced or metastatic pancreatic cancer. The study was powered to detect a $33 \%$ difference in OS and 569 patients were enrolled. The first 521 patients were randomized to erlotinib $100 \mathrm{mg}$ per day or placebo; the remainder received erlotinib $150 \mathrm{mg}$ per day or placebo. The study was reported using the log rank adjusted for performance status and extent of disease. Although the difference in median OS between the erlotinib and placebo groups was modest (6.24 months vs 5.91 months), there was a significant decrement in the hazard ratio for death (0.82; 95\% CI, 0.69-0.99; $P=0.04)$ and a significant improvement in 1 -year survival rate $(23 \%$ vs $17 \%, P=0.02)$. In this trial, $72 \%$ of the 282 erlotinib treated patients developed a rash. ${ }^{3}$

An additional small, phase II trial has demonstrated promising activity from the addition of erlotinib to cytotoxic chemotherapy in the second line. The combination of erlotinib (150 mg daily) and capecitabine $\left(1000 \mathrm{mg} / \mathrm{m}^{2}\right.$, twice daily for 14 days, every 3 weeks) was examined in 30 patients with gemcitabine-refractory, metastatic disease. A $10 \%$ overall response rate by RECIST criteria was seen. The median survival duration was 6.5 months, similar to results of other published phase II trials of second line therapy. ${ }^{22}$

Based on the NCIC CTG trial results, the Food and Drug Administration (FDA) and the European Medicines Evaluation Agency (EMEA) have approved the combination of erlotinib and gemcitabine for first-line treatment of advanced pancreatic cancer. An informal, post hoc analysis performed in 2007 estimated the incremental cost-effectiveness ratio for the addition of erlotinib at US\$410,000 per year of life saved. When adjusted for the quality-of-life impact of diarrhea, this number rose to US\$430,000 to US\$510,000 per quality adjusted life year. ${ }^{23}$ This has brought into question the clinical significance of erlotinib's small survival benefit. A consensus has not yet been reached on the addition of erlotinib in the treatment of unselected patients with advanced disease.

The combination of gefitinib and gemcitabine has also been studied in a small, single-arm, phase II study in Europe, by the Hellenic Cooperative Oncology Group. Fifty-three patients with inoperable or metastatic pancreatic cancer were treated with gefitinib (250 mg per day) and gemcitabine (1000 mg/m², weekly for 7 weeks, followed by a 1 week rest, then $1000 \mathrm{mg} / \mathrm{m}^{2}$ weekly for 3 of every 4 weeks). The 6 month progression-free survival (PFS) was 30\%, with a median PFS of 4.1 months. The 1 -year survival rate was $27 \%$, with a median survival of 7.3 months. ${ }^{24}$ These results are comparable to those from the erlotinib and gemcitabine arm in the NCIC CTG trial, but have not been confirmed in a randomized trial.

\section{EGFR-directed antibody therapy in advanced pancreatic cancer}

EGFR-directed antibody therapy has also been investigated in pancreatic cancer. Cetuximab was first studied in combination with gemcitabine in a phase II study of chemotherapynaïve patients with advanced disease. Forty-one patients with EGFR immunostain-positive tumors (6 locally advanced, 34 metastatic) were treated with cetuximab (initial dose $400 \mathrm{mg} / \mathrm{m}^{2}$, followed by $250 \mathrm{mg} / \mathrm{m}^{2}$ weekly) and gemcitabine (1000 mg/m², weekly for 7 weeks, followed by a 1 week rest, then $1000 \mathrm{mg} / \mathrm{m}^{2}$, weekly for 3 weeks, every 4 weeks). Five patients $(12.5 \%)$ achieved a partial response and 26 patients $(63.4 \%)$ had stable disease. The median time to progression was 3.8 months, and the median OS duration was 7.1 months. The 1-year survival rate of $31.7 \%$ compared favorably to $18 \%$ with gemcitabine alone in historical series. The combination was well tolerated with the most commonly reported toxicities of all grades as follows: rash (87.8\%), nausea $(61.0 \%)$, weight loss $(58.5 \%)$, and diarrhea $(53.7 \%) .{ }^{25}$

Subsequent to these seemingly promising results, two randomized trials have been presented that demonstrated no significant survival benefit from the addition of cetuximab to cytotoxic chemotherapy for advanced disease. The Southwest Oncology Group (SWOG) S0205 study was a randomized phase III trial of gemcitabine with or without cetuximab in patients with locally advanced or metastatic pancreatic adenocarcinoma in 766 patients. The data, presented in 2007, demonstrated a nonsignificant difference in PFS; 3 vs 3.5 months $(P=0.14)$, and OS duration; 6 vs 6.5 months $(P=0.058)$, in the gemcitabine alone vs gemcitabine and cetuximab groups, respectively. ${ }^{26}$ It should be noted that although these results were not statistically significant in this trial, numerically they are similar to the results in the control and experimental arms of the NCIC CTG trial of gemcitabine with or without erlotinib.

The second study was a randomized phase II trial conducted by the Eastern Cooperative Oncology Group (ECOG), which randomized 87 eligible patients with metastatic pancreatic cancer to the Murren regimen of docetaxel and irinotecan with or without cetuximab. ${ }^{27,28}$ Updated results were presented in 2008. The study was not powered for comparison of the two arms, and all comparisons are exploratory. Unexpectedly, the patients treated with 
cetuximab had a shorter median survival duration of 5.3 months compared to 6.5 months for the placebo group. A very short post-progression survival was observed in the cetuximab arm, not well explained by the data. Possible explanations include low utilization of second line chemotherapy, related either to the poor performance status of the patients at time of progression, or to persistent toxicities from the regimen, or an acceleration of tumor growth after withdrawal of cetuximab. The toxicity of this regimen was high, even without cetuximab. Grade 3 or 4 diarrhea was observed in $47 \%$ of patients in the three drug arm and the rate of treatment-related death was $4 \%{ }^{29}$

\section{EGFR- and VEGF-targeted therapy combinations in advanced disease}

Following up on the NCIC CTG trial of erlotinib and gemcitabine, Van Cutsem and colleagues evaluated the benefit of this regimen combined with the vascular endothelial growth factor inhibitor, bevacizumab. In their trial, 607 chemotherapy-naïve patients with metastatic disease were randomized to receive placebo or bevacizumab
( $5 \mathrm{mg} / \mathrm{kg}$ every 2 weeks) in combination with gemcitabine (1000 mg/m², weekly for 7 weeks, followed by a 1 week rest, then $1000 \mathrm{mg} / \mathrm{m}^{2}$ weekly for 3 weeks, every 4 weeks) and erlotinib (100 mg/m² daily). At the final analysis, 454 patients had died (233 in the placebo arm; 221 in the bevacizumab arm). The median OS was not significantly different between the two groups (6.0 months for the placebo arm vs 7.1 months in the bevacizumab arm [HR 0.89; 95\% CI 0.74-1.07]); however the median PFS was significantly longer for the bevacizumab-treated patients (3.6 months for the placebo arm vs. 4.6 months for the bevacizumab arm [HR $0.73 ; 95 \%$ CI $0.61-0.86 ; P<0.01]) .{ }^{30}$ The lack of statistical significance of the OS benefit may be due to the low power of this study.

Additional phase II trials have examined the combination of EGFR- and VEGF-targeted therapy in advanced pancreatic cancer. A multicenter, phase II trial was conducted that randomized 139 evaluable patients with advanced disease to receive either weekly cetuximab at standard doses or erlotinib (150 mg/m² $\mathrm{d} 1-5,8-12$, and $15-26$ of a 28 -day cycle) in addition to gemcitabine and bevacizumab. There were no

Table I Summary of studies of EGFR targeted therapies for advanced pancreatic cancer

\begin{tabular}{|c|c|c|c|c|c|}
\hline Study treatment & Phase & Pts & $\begin{array}{l}\text { Med OS } \\
\text { (mos) }\end{array}$ & $\begin{array}{l}\text { Med PFS } \\
\text { (mos) }\end{array}$ & $\begin{array}{l}\text { Rash } \\
\text { [Gr3-4] (\%) }\end{array}$ \\
\hline \multicolumn{6}{|l|}{ EGFR targeted therapy } \\
\hline Moore $2007^{3}$ & III & & & & \\
\hline Gemcitabine + Placebo & & 284 & 5.91 & 3.55 & $29[1]$ \\
\hline Gemcitabine + Erlotinib & & 285 & 6.24 & 3.75 & $72[6]$ \\
\hline Fountzilas $2008^{24}$ & II & & & & \\
\hline Gemcitabine + Gefitinib & & 53 & 7.3 & 4.1 & $56[4]$ \\
\hline Xiong $2004^{25}$ & II & & & & \\
\hline Gemcitabine + Cetuximab & & 41 & 7.1 & 3.8 & $88[12]$ \\
\hline Philip $2007^{26}$ & III & & & & \\
\hline Gemcitabine & & 369 & 6 & 3 & NR [0] \\
\hline Gemcitabine + Cetuximab & & 366 & 6.5 & 3.5 & NR [7.I] \\
\hline Burtness $2007^{28}$ & II & & & & \\
\hline Irinotecan, Docetaxel & & 46 & 6.5 & 3.9 & $9[0]$ \\
\hline Irinotecan, Docetaxel + Cetuximab & & 45 & 5.3 & 4.5 & $67[0]$ \\
\hline \multicolumn{6}{|c|}{ Combined EGFR and VEGF targeted therapy } \\
\hline Van Cutsem $2009^{30}$ & III & & & & \\
\hline Gemcitabine, Erlotinib + Placebo & & 301 & 6 & 3.6 & $44[3]$ \\
\hline Gemcitabine, Erlotinib + Bevacizumab & & 306 & 7.1 & 4.6 & $49[8]$ \\
\hline Kindler $2008^{31}$ & II & & & & \\
\hline Gemcitabine, Bevacizumab + Cetuximab & & 68 & 7.8 & 5.0 & NR $[10]$ \\
\hline Gemcitabine, Bevacizumab + Erlotinib & & 71 & 7.2 & 5.1 & NR [6] \\
\hline Ko $2009^{32}$ & II & & & & \\
\hline Bevacizumab, Cetuximab & & 29 & NR & 1.8 & $58.6[N R]$ \\
\hline Bevacizumab, Cetuximab + Gemcitabine & & 28 & NR & 3.5 & $28.6[N R]$ \\
\hline
\end{tabular}

Abbreviations: EGFR, epidermal growth factor receptor; Gr, grade; mos, months; NR, not reported; OS, overall survival; PFS, progression-free survival; VEGF, vascular endothelial growth factor. 
statistically significant differences between the two groups in median OS (7.8 months vs 7.1 months for the cetuximab and erlotinib groups, respectively), both of which were superior to historical controls of gemcitabine monotherapy. ${ }^{31}$

Finally, an open-label, phase II, randomized trial examined the benefit of cetuximab and bevacizumab $(10 \mathrm{mg} / \mathrm{kg}$ every 2 weeks) either alone or in combination with weekly gemcitabine. A total of 57 chemotherapy-naïve patients with advanced disease were randomized between the two groups. The combination of the two antibodies alone resulted in no objective responses and a median PFS of 1.8 months compared to 3.5 months with the addition of gemcitabine. ${ }^{32}$ It seems that the use of the two antibodies alone is inferior to gemcitabine-based therapy.

\section{EGFR-directed therapy in combination with radiation for locally advanced disease}

The role of EGFR-directed therapy in combination with radiation therapy is under active investigation. Preclinical data have demonstrated that the addition of cetuximab or erlotinib to the combination of gemcitabine and radiation inhibits pancreatic cell growth significantly more than gemcitabine and radiation alone. ${ }^{12} \mathrm{~A}$ Phase 1 trial examined the combination of weekly gemcitabine $\left(300 \mathrm{mg} / \mathrm{m}^{2}\right)$, cetuximab (400 mg/m² loading dose, then $250 \mathrm{mg} / \mathrm{m}^{2}$ ), and escalating doses of radiation in 25 patients with locally advanced disease. The combined therapy was well tolerated with no grade 4 toxicity reported and a maximum tolerated dose was not reached. Typical skin rash was observed in 19 patients (76\%). Of the 20 evaluable patients, one demonstrated radiological complete response, 8 partial responses, and 6 patients had stable disease. ${ }^{33}$

Final results of a phase II trial examining the addition of cetuximab to gemcitabine and intensity modulated radiotherapy (IMRT) in patients with locally advanced, inoperable pancreatic cancer were recently presented. ${ }^{34}$ Sixty-eight chemotherapy-naïve patients received IMRT with weekly gemcitabine $\left(300 \mathrm{mg} / \mathrm{m}^{2}\right)$ and cetuximab (400 mg/m² loading dose, then $250 \mathrm{mg} / \mathrm{m}^{2}$ ). Patients were randomized following completion of radiotherapy to weekly gemcitabine alone $\left(1000 \mathrm{mg} / \mathrm{m}^{2}\right)$ for 4 weeks, or weekly gemcitabine $\left(1000 \mathrm{mg} / \mathrm{m}^{2}\right)$ and cetuximab $\left(250 \mathrm{mg} / \mathrm{m}^{2}\right)$ over 12 weeks. All patients were subsequently treated with weekly gemcitabine alone. One patient had a complete response, 23 had partial responses, 41 had stable disease, and only 3 had progressive disease. Fourteen patients went on to have complete surgical resections. For all patients, the 1 - and 2-year OS were $61 \%$ and $20 \%$, respectively, with no significant differences between the two study groups. Median survival was 15 months. For comparison, in the recently presented ECOG trial, E4201, patients with locally advanced disease who received gemcitabine and IMRT had 1 - and 2-year OS of $50 \%$ and $12 \%$, respectively. Median survival in E4201 was 11 months. ${ }^{35}$ Although these results appear promising, a randomized trial comparing cetuximab, gemcitabine, and radiation to gemcitabine and radiation has yet to be performed, and may not be justified in view of the negative trials in the metastatic setting.

\section{Rash as a marker of efficacy}

In multiple trials of EGFR-targeted therapies, a higher grade of rash has correlated with improved outcome. In the NCIC CTG trial of gemcitabine with or without erlotinib, median survival was significantly longer for the erlotinib-treated patients with grade 2 or greater rash compared to those with grade 1 or 0 (10.5 months, 5.8 months, and 5.3 months, respectively $[P=0.037])$. This may be explained, in part, by the association of skin rash in this trial with age younger than 65 years $(P=0.01)$ and good performance status $(P=0.03){ }^{3}$

As in the NCIC CTG trial, a relationship between treatment efficacy and rash was suggested in the trial by Vervenne and colleagues of gemcitabine and erlotinib with bevacizumab or placebo. In the placebo arm, the 77 patients who experienced a grade 2 or higher rash had a median OS of 8.3 months compared to 4.3 months for the 123 patients who did not develop a rash. Results were similar in the bevacizumab arm (8.4 months vs 5.0 months). Neither difference met statistical significance. ${ }^{36}$

A similar association was demonstrated for cetuximab in the phase II trial of its combination with gemcitabine. The median OS in patients correlated with grade of rash as follows: 2.3 months for no rash, 5.7 months for grade 1, 8.3 months for grade 2, and 13.9 months for grade 3 . This association between rash grade and median OS was statistically significant $(P<0.01){ }^{25}$

The etiology and clinical significance of this association remain to be determined. Rash may serve as a marker of host immunocompetency in that it signifies an ability to mount an inflammatory response. Alternatively, it has been postulated that variability in drug absorption or metabolism may be responsible; those patients who obtain a higher or more sustained blood level of drug being more likely to inhibit dermal EGFR and develop a rash. In support, 
gefitinib trough levels have been found to be highly variable amongst patients in clinical trials. ${ }^{37}$ Furthermore, higher trough levels of erlotinib have been associated with the severity of rash in patients treated for NSCLC. ${ }^{38}$ Genetic polymorphisms in the ABCG2 transporter protein, which is involved in intestinal absorption and hepatic elimination of gefitinib and erlotinib, have been found to contribute to inter-patient differences in bioavailability and pharmacokinetics. ${ }^{38,39}$ Additionally, genetic differences in the cytochromes involved in their hepatic metabolism, such as CYP3A4/5, are partly responsible. ${ }^{18,37}$

The association between drug levels and rash has not been demonstrated, however, for the monoclonal antibodies, although in diseases in which cetuximab significantly delays progression or death, skin rash is also a positive predictive marker. A phase 1 escalating-dose trial of weekly cetuximab given to patients with epithelial malignancies found no association between cetuximab trough levels and the development of rash. Cetuximab at doses greater than $250 \mathrm{mg} / \mathrm{m}^{2}$ did, however, lead to a dose-dependent decrease in EGFR protein expression levels in skin over time. ${ }^{40}$ Additionally, a trial of dose escalation in patients who did not develop significant rash to cetuximab at standard doses was performed. Patients who had less than a grade 2 rash at standard doses of cetuximab $\left(250 \mathrm{mg} / \mathrm{m}^{2}\right)$ were randomized to receive either standard dose cetuximab or escalating doses until a grade 2 or higher rash developed or the maximum dose of $500 \mathrm{mg} / \mathrm{m}^{2}$ was reached. Contrary to the hypothesis, there was no difference in rash incidence between the two groups. ${ }^{41}$

An alternative explanation for the association between rash and efficacy of EGFR targeted therapy may involve genetic variability affecting the expression of EGFR in both germline and tumor cells. A genetic polymorphism in EGFR expression involving a dinucleotide repeat polymorphism in EGFR intron 1 has been described. This CA repeat is located upstream to an EGFR enhancer region and in vitro data have shown an inverse correlation between the number of CA repeats and EGFR transcription. ${ }^{42}$ This polymorphism was prospectively associated with the development of rash from gefitinib therapy in patients with lung cancer. ${ }^{43}$ Whether this polymorphism correlates with response to EGFR TKI therapy in pancreatic cancer is unknown.

\section{Limitations of EGFR-targeted therapy}

Although EGFR is a frequently expressed target that plays an active role in pancreatic adenocarcinoma carcinogenesis, the efficacy of EGFR targeted therapy has not met expectations.
There are several proposed mechanisms that relate to both limitations of the agents tested to date and to the biological environment of pancreatic cancer.

First, monoclonal antibodies, such as cetuximab and panitumimab, are large molecules that may not distribute well to or within pancreatic tumors. Optimal dosing for these agents may not be known and may dependent on EGFR content, ligand levels, or other not yet identified variables. As mentioned above, the development of rash is associated with treatment efficacy of these agents and correlates with trough levels of the EGFR TKIs, suggesting that pharmacogenetic differences in absorption and metabolism may lead to subtherapeutic blood levels in a proportion of patients.

Furthermore, EGFR TKIs have not resulted in any sustained tumor responses in pancreatic cancer trials, as they have in a proportion of patients with NSCLC. This is likely due to differences in the prevalence of activating mutations in EGFR that have been associated with these prolonged responses in NSCLC. ${ }^{44}$ In four small case series, activating EGFR gene mutations were identified in only 2 out of $170(1.2 \%)$ specimens of pancreatic cancer. ${ }^{45-48}$

\section{Mechanisms of resistance to EGFR targeted therapy}

Above all, a high rate of acquired or inherent resistance of pancreatic cancer to EGFR targeted therapy is most likely to explain the lack of efficacy. Multiple potential mechanisms of EGFR targeted therapy have been well described. ${ }^{49-51}$

The most well-defined mechanisms of resistance involve EGFR independent activation of downstream mediators. Activating mutations of $K R A S$, for example, have been examined retrospectively in several clinical trials and have been associated with a lack of response to or survival benefit from EGFR-targeted antibodies in colorectal cancer, as well as a lack of response to EGFR TKI's in NSCLC. ${ }^{52-54}$ In pancreatic cancer, KRAS mutations are prevalent, found in 50 to $95 \%$ of tumor samples in multiple series. ${ }^{5,6}$ The relationship with KRAS mutations and efficacy of EGF-targeted therapies in this disease has yet to be established, but can be inferred as a likely mechanism of resistance for a large proportion of patients.

Another example of resistance due to constitutive activation of a downstream mediator involves PI3K-Akt. Gefitinib-resistant cell lines of squamous cell cancer developed in cell culture maintain activity of PI3K-Akt despite demonstrable inhibition of EGFR. The increased PI3K-Akt activity in these cells is due to increased activity of an 
alternative pathway, the insulin-like growth factor 1 receptor (IGF1R) pathway. Down-regulation of the insulin-like growth factor binding protein has been implemented in this process. Combined inhibition of both EGFR and IGF1R with both gefitinib and a targeted anti-IGF1R TKI is effective in inhibiting both PI3K-Akt activity and cell growth in cell lines. This has been verified in animal models, as well. In mice bearing xenografts of squamous cell carcinoma, treatment with gefitinib alone led to reduction in tumor size, though most tumors ultimately progressed on this therapy. Treatment with an anti-IGF1R monoclonal antibody alone led only to minor and short-lived partial responses. Combined therapy with both gefinitib and the anti-IGF1R antibody, however, led to complete responses in all of the treated mice that persisted for several months after the discontinuation of therapy. ${ }^{55}$

In light of these promising preclinical data, the Southwest Oncology Group has begun enrolling patients to a combination phase I/randomized phase II trial comparing gemcitabine and erlotinib therapy with or without the addition of an IGF1R antibody, IMC-A12, in chemo-naïve patients with metastatic pancreatic cancer. Study completion in 2011 is anticipated.

An alternative mechanism of EGFR resistance via increased PI3K-Akt activity involves PTEN, a lipid phosphatase and tumor suppressor protein. Loss of PTEN (often through loss of one allele and mutation in the other) leads to accumulation of its major substrate, phosphatidylinositol 3,4,5-triphosphate, which binds and activates Akt. ${ }^{56,57}$ PTEN loss has been associated with resistance to cetuximab therapy in a small study of 27 patients with metastatic colon cancer. ${ }^{58}$ PTEN mutations also result in gefitinib resistance in breast cancer cells lines. Gefinitib therapy was associated with upregulation of PI3k-Akt in these PTEN mutant cells. Furthermore, restoration of wild type PTEN via an adenoviral vector led to decreased activity of the PI3K-Akt pathway and improved response of these cells to gefitinib supporting unopposed PI3K-Akt activity as the mechanism of resistance..$^{59}$

In NSCLC, EGFR TKI resistance has also been attributed to persistent activity of PI3K-Akt, but through yet another mechanism. Amplification of MET, a protooncogene that encodes a transmembrane tyrosine kinase receptor for the hepatocyte growth factor (scatter factor) has been described as a mechanism of acquired resistance to gefitinib in NSCLC cell lines. MET amplification leads to EGFR-independent activation of PI3K-Akt through activation of erbB-3-dependent signaling. MET targeted therapy alone is ineffective at overcoming this resistance, but in combination with gefitinib tumor cell death has been achieved. Furthermore, examination of tumor samples from patients who have developed resistance to EGFR TKI therapy has shown emergence of MET gene amplification that was not present in tumor samples prior to the initiation of the treatment. $^{60}$

Therapies that directly inhibit P13K-Akt have been developed, as well. In mice bearing gefinitib-resistant NSCLC xenografts, the PI3K inhibitor PX-866 was shown to enhance the anti-tumor effect of gefitinib. Tumor growth, however, slowly increased towards the end of the treatment period, suggesting emergence of yet additional resistance pathways. The major toxicity of this agent was reversible hyperglycemia that resulted from a target-related decrease in insulin sensitivity. ${ }^{61}$

Additionally, efforts to restore sensitivity to anti-EGFR resistant tumor cells have focused on targeting a downstream mediator of the PI3K-Akt pathway: the mammalian target of rapamycin (mTOR). mTOR is a serine/threonine-specific kinase responsible for mitogen-induced cell proliferation and survival signaling. ${ }^{62,63}$ mTOR is thought to integrate proliferative, antiapoptotic, and antiogenic signaling by connecting VEGF, hypoxia-inducible factor 1 (HIF-1) and multiple ErbB family receptor pathways. The mTOR inhibitor, everolimus, has been studied in combination with EGFR targeted therapies in a variety of resistant tumor cell lines. In combination with cetuximab or gefitinib, everolimus resulted in strong reduction of PI3K-Akt activity. It also restored anti-EGFR therapy growth inhibition of resistant cell lines in a dose-dependent fashion. Of particular interest, gefitinib efficacy was restored when given with everolimus to resistant prostate cancer cells harboring an inactivating PTEN mutation. ${ }^{64}$ Phase one clinical trials of therapies targeting both mTOR and EGFR, such as everolimus and cetuximab, are currently enrolling patients.

Finally, resistance to TKI therapy can develop through conformational alterations in the ATP binding domain of the EGFR. As previously described, known mutations in the EGFR gene result in increased activity of the receptor and are associated with an improved response to EGFR TKI therapy. Contrarily, a point mutation in the EGFR gene, which results in the substitution of methionine for threonine at position 790 (T790M), decreases the ability of erlotinib or gefitinib to inhibit EGFR. These mutations have been identified in approximately $50 \%$ of lung adenocarcinoma samples from patients who had acquired resistance to EGFR TKI therapy. ${ }^{65-67}$ The mutation is thought to result in resistance by altering the structure of the ATP-binding pocket in the 
tyrosine kinase domain. This alteration leads to increased affinity for ATP, which can then out-compete gefitinib and/or erlotinib for binding to the receptor. Irreversible inhibitors of EGFR tyrosine kinase are currently in development that form permanent covalent bonds to the binding domain rather than having to compete with ATP for temporary interactions. They have been shown to successfully inhibit EGFR bearing the T790M mutation in vitro. ${ }^{50}$

\section{Conclusions}

Erlotinib is the only EGFR-targeted therapy currently approved by the FDA for use (in combination with gemcitabine) in the management of advanced pancreatic cancer. The clinical significance of its additional benefit has been appropriately questioned. ${ }^{23}$ The identification of biological factors that predict benefit is needed to improve patient selection and thereby the utility of these drugs. Similarly, prospective research is warranted to determine whether the development of rash should act as a correlate for their efficacy.

Pancreatic cancer appears to have high rates of resistance to EGFR-targeted therapies through several mechanisms that include receptor-independent activation of downstream mediators. Our ability to overcome resistance mechanisms will ultimately determine the success of EGFR-targeted therapies in this malignancy. Several approaches have had success in preclinical models. We await the results of their application in clinical trials.

\section{Disclosure}

Dr. Faller declares no conflicts of interest. Dr. Burtness reports research funding and consulting fees from Genentech.

\section{References}

1. Parkin DM, Bray F, Ferlay J, Pisani P. Global cancer statistics, 2002. CA Cancer J Clin. 2005;55:74-108.

2. Burris HA 3rd, Moore MJ, Andersen J, et al. Improvements in survival and clinical benefit with gemcitabine as first-line therapy for patients with advanced pancreas cancer: a randomized trial. J Clin Oncol. 1997; 15:2403-2413.

3. Moore MJ, Goldstein D, Hamm J, et al. Erlotinib plus gemcitabine compared with gemcitabine alone in patients with advanced pancreatic cancer: a phase III trial of the National Cancer Institute of Canada Clinical Trials Group. J Clin Oncol. 2007;25:1960-1966.

4. Grant S, Fisher PB, Dent P. The role of signal transduction pathways in drug and radiation resistance. Cancer Treat Res. 2002;112: 89-108.

5. Smit VT, Boot AJ, Smits AM, Fleuren GJ, Cornelisse CJ, Bos JL. KRAS codon 12 mutations occur very frequently in pancreatic adenocarcinomas. Nucleic Acids Res. 1988;16:7773-7782.

6. Almoguera C, Shibata D, Forrester K, Martin J, Arnheim N, Perucho M. Most human carcinomas of the exocrine pancreas contain mutant c-K-ras genes. Cell. 1988;53:549-554.

7. Deramaudt T, Rustgi AK. Mutant KRAS in the initiation of pancreatic cancer. Biochim Biophys Acta. 2005;1756:97-101.
8. Fjallskog ML, Lejonklou MH, Oberg KE, Eriksson BK, Janson ET. Expression of molecular targets for tyrosine kinase receptor antagonists in malignant endocrine pancreatic tumors. Clin Cancer Res. 2003;9: 1469-1473.

9. Tobita K, Kijima H, Dowaki S, et al. Epidermal growth factor receptor expression in human pancreatic cancer: Significance for liver metastasis. Int J Mol Med. 2003;11:305-309.

10. Ueda S, Ogata S, Tsuda $H$, et al. The correlation between cytoplasmic overexpression of epidermal growth factor receptor and tumor aggressiveness: poor prognosis in patients with pancreatic ductal adenocarcinoma. Pancreas. 2004;29:e1-e8.

11. Bloomston M, Bhardwaj A, Ellison EC, Frankel WL. Epidermal growth factor receptor expression in pancreatic carcinoma using tissue microarray technique. Dig Surg. 2006;23:74-79.

12. Morgan MA, Parsels LA, Kollar LE, Normolle DP, Maybaum J, Lawrence TS. The combination of epidermal growth factor receptor inhibitors with gemcitabine and radiation in pancreatic cancer. Clin Cancer Res. 2008;14:5142-5149.

13. Bruns CJ, Solorzano CC, Harbison MT, et al. Blockade of the epidermal growth factor receptor signaling by a novel tyrosine kinase inhibitor leads to apoptosis of endothelial cells and therapy of human pancreatic carcinoma. Cancer Res. 2000;60:2926-2935.

14. Ng SS, Tsao MS, Nicklee T, Hedley DW. Effects of the epidermal growth factor receptor inhibitor OSI-774, Tarceva, on downstream signaling pathways and apoptosis in human pancreatic adenocarcinoma. Mol Cancer Ther. 2002;1:777-783.

15. Friess H, Berberat P, Schilling M, Kunz J, Korc M, Buchler MW. Pancreatic cancer: the potential clinical relevance of alterations in growth factors and their receptors. J Mol Med. 1996;74:35-42.

16. Feng J, Adsay NV, Kruger M, et al. Expression of ERRP in normal and neoplastic pancreata and its relationship to clinicopathologic parameters in pancreatic adenocarcinoma. Pancreas. 2002;25:342-349.

17. Ciardiello F, Tortora G. EGFR antagonists in cancer treatment. $N$ Engl J Med. 2008;358:1160-1174.

18. Li J, Zhao M, He P, Hidalgo M, Baker SD. Differential metabolism of gefitinib and erlotinib by human cytochrome P450 enzymes. Clin Cancer Res. 2007;13:3731-3737.

19. Lynch TJ, Bell DW, Sordella R, et al. Activating mutations in the epidermal growth factor receptor underlying responsiveness of nonsmall-cell lung cancer to gefitinib. $N$ Engl J Med. 2004;350:2129-2139.

20. Chung CH, Mirakhur B, Chan E, et al. Cetuximab-induced anaphylaxis and IgE specific for galactose-alpha-1,3-galactose. $N$ Engl J Med. 2008;358:1109-1117.

21. Baselga J, Pfister D, Cooper MR, et al. Phase I studies of antiepidermal growth factor receptor chimeric antibody C225 alone and in combination with cisplatin. J Clin Oncol. 2000;18:904-914.

22. Oh D, Lee K, Lee K, et al. A phase II trial of erlotinib in combination with gemcitabine and capecitabine in previously untreated metastatic/ recurrent pancreatic cancer. J Clin Oncol. 2008;26(May 20 Suppl): Abstract 4638.

23. Miksad RA, Schnipper L, Goldstein M. Does a statistically significant survival benefit of erlotinib plus gemcitabine for advanced pancreatic cancer translate into clinical significance and value? J Clin Oncol. 2007;25:4506-4507; author reply 8.

24. Fountzilas G, Bobos M, Kalogera-Fountzila A, et al. Gemcitabine combined with gefitinib in patients with inoperable or metastatic pancreatic cancer: a phase II Study of the Hellenic Cooperative Oncology Group with biomarker evaluation. Cancer Invest. 2008;26: 784-793.

25. Xiong HQ, Rosenberg A, LoBuglio A, et al. Cetuximab, a monoclonal antibody targeting the epidermal growth factor receptor, in combination with gemcitabine for advanced pancreatic cancer: a multicenter phase II Trial. J Clin Oncol. 2004;22:2610-2316.

26. Philip PA, Benedetti J, Fenoglio-Presier C, et al. Phase III study of gemcitabine $[\mathrm{G}]$ plus cetuximab $[\mathrm{C}]$ versus gemcitabine in patients [pts] with locally advanced or metastatic pancreatic adenocarcinoma [PC]: SWOG S0205 study. J Clin Oncol. 2007;25:LBA4509. 
27. Bleickardt E, Argiris A, Rich R, et al. Phase I dose escalation trial of weekly docetaxel plus irinotecan in patients with advanced cancer. Cancer Biol Ther. 2002;1:646-651.

28. Burtness B, Thomas L, Sipples R, et al. Phase II trial of weekly docetaxel/irinotecan combination in advanced pancreatic cancer. Cancer J. 2007;13:257-262.

29. Burtness BA, Powell ME, Berlin D, et al. Phase II ECOG trial of irinotecan/docetaxel with or without cetuximab in metastatic pancreatic cancer: Updated survival and CA19-9 results. J Clin Oncol. 2008;26(May 20 Suppl): Abstract 4642.

30. Van Cutsem E, Vervenne WL, Bennouna J, et al. Phase III trial of bevacizumab in combination with gemcitabine and erlotinib in patients with metastatic pancreatic cancer. J Clin Oncol. 2009;27:2231-2237.

31. Kindler HL, Gangadhar T, Karrison T, et al. Final analysis of a randomized phase II study of bevacizumab and gemcitabine plus cetuximab or erlotinib in patients with advanced pancreatic cancer. J Clin Oncol. 2008;26(May 20 Suppl): Abstract 4502.

32. Ko AH, Dicke K, Gurtler J, et al. Phase II, randomized, open-label study of cetuximab and bevacizumab alone or in combination with fixed-dose rate gemcitabine as first-line therapy for patient with metastatic adenocarcinoma of the pancreas. In: Gastrointestinal Cancers Symposium; 2009 January 15-17; San Francisco, CA; 2009. Abstract 183.

33. Demols A, Mahin C, Marechal R, et al. Cetuximab plus chemoradiation combined therapy for locally advanced inoperable pancreatic adenocarcinoma: A phase I study. J Clin Oncol. 2008;26(May 20 Suppl): Abstract 4629.

34. Munter M, Timke C, Adbollahi A, et al. Final results of a phase II trial [PARC-Study ISRCTN56652283] for patients with primary inoperable locally advanced pancreatic cancer combining intensity modulated radiotherapy (IMRT) with cetuximab and gemcitabine. J Clin Oncol. 2008;26(May 20 Suppl): Abstract 4613.

35. Loehrer PJ, Powell ME, Cardenes HR, et al. A randomized phase III study of gemcitabine in combination with radiation therapy versus gemcitabine alone in patients with localized, unresectable pancreatic cancer: E4201. J Clin Oncol. 2008;26(May 20 Suppl): Abstract 4506.

36. Van Cutsem E, Vervenne WL, Bennouna J, Humblet Y, Cosaert J. Rash as a marker for the efficacy of gemcitabine plus erlotinib-based therapy in pancreatic cancer: Results from the AViTA study. In: Gastrointestinal Cancer Symposium; 2009 January 15-17; San Franciso, CA; 2009. Abstract 117.

37. Li J, Karlsson MO, Brahmer J, et al. CYP3A phenotyping approach to predict systemic exposure to EGFR tyrosine kinase inhibitors. J Natl Cancer Inst. 2006;98:1714-1723.

38. Rudin CM, Liu W, Desai A, et al. Pharmacogenomic and pharmacokinetic determinants of erlotinib toxicity. J Clin Oncol. 2008;26:1119-1127.

39. Li J, Cusatis G, Brahmer J, et al. Association of variant ABCG2 and the pharmacokinetics of epidermal growth factor receptor tyrosine kinase inhibitors in cancer patients. Cancer Biol Ther. 2007;6:432-438.

40. Fracasso PM, Burris H 3rd, Arquette MA, et al. A phase 1 escalating single-dose and weekly fixed-dose study of cetuximab: pharmacokinetic and pharmacodynamic rationale for dosing. Clin Cancer Res. 2007;13:986-993.

41. Tejpar S, Peeters M, Humblet Y, et al. Dose-escalation study using up to twice the standard dose of cetuximab in patients with metastatic colorectal cancer (mCRC) with no or slight skin reactions on cetuximab standard dose treatment (EVEREST study): Preliminary data. J Clin Oncol. 2006;24:3554.

42. Gebhardt F, Zanker KS, Brandt B. Modulation of epidermal growth factor receptor gene transcription by a polymorphic dinucleotide repeat in intron 1. J Biol Chem. 1999;274:13176-13180.

43. Huang CL, Yang CH, Yeh KH, et al. EGFR intron 1 dinucleotide repeat polymorphism is associated with the occurrence of skin rash with gefitinib treatment. Lung Cancer. 2009;64:346-351.

44. Sequist LV, Martins RG, Spigel D, et al. First-line gefitinib in patients with advanced non-small-cell lung cancer harboring somatic EGFR mutations. J Clin Oncol. 2008;26:2442-2449.
45. Immervoll H, Hoem D, Kugarajh K, Steine SJ, Molven A. Molecular analysis of the EGFR-RAS-RAF pathway in pancreatic ductal adenocarcinomas: lack of mutations in the BRAF and EGFR genes. Virchows Arch. 2006;448:788-796.

46. Iyer RV, Rossi M, Bharthuar M, et al. Evaluation of phosphatidyl inositol 3-kinase catalytic subunit (PI3KCA) and epidermal growth factor receptor (EGFR) gene mutations in pancreatic adenocarcinoma. In: Gastrointestinal Cancers Symposium January 2009; San Francisco, CA; 2009. Abstract 173.

47. Kwak EL, Jankowski J, Thayer SP, et al. Epidermal growth factor receptor kinase domain mutations in esophageal and pancreatic adenocarcinomas. Clin Cancer Res. 2006;12:4283-4287.

48. Morinaga S, Sugano N, Wada H, et al. Epidermal growth factor receptor (EGFR) status: Mutation and protein expression, and clinicopathology of pancreatic ductal adenocarcinoma. In: Gastrointestinal Cancers Symposium; 2008 January 25-27; Orlando, FL; 2008. Abstract 185.

49. Camp ER, Summy J, Bauer TW, Liu W, Gallick GE, Ellis LM. Molecular mechanisms of resistance to therapies targeting the epidermal growth factor receptor. Clin Cancer Res. 2005;11:397-405.

50. Dempke WC, Heinemann V. Resistance to EGF-R (erbB-1) and VEGF-R modulating agents. Eur J Cancer. 2009. In press.

51. Rexer BN, Engelman JA, Arteaga CL. Overcoming resistance to tyrosine kinase inhibitors: lessons learned from cancer cells treated with EGFR antagonists. Cell Cycle. 2009;8:18-22.

52. Bokemeyer C, Bondarenko I, Hartmann JT, et al. KRAS status and efficacy of first-line treatment of patients with metastatic colorectal cancer (mCRC) with FOLFOX with or without cetuximab: The OPUS experience. J Clin Oncol. 2008;26(May 20 Suppl): Abstract 4000.

53. Jackman DM, Sequist LV, Cioffredi L, et al. Impact of EGFR and KRAS genotype on outcomes in a clinical trial registry of NSCLC patients initially treated with erlotinib or gefitinib. J Clin Oncol. 2008; 26(May 20 Suppl): Abstract 8035.

54. Van Cutsem E, Lang I, D'haens G, et al. KRAS status and efficacy in the first-line treatment of patients with metastatic colorectal cancer (mCRC) treated with FOLFIRI with or without cetuximab: The CRYSTAL experience. J Clin Oncol. 2008;26(May 20 Suppl): Abstract 2.

55. Guix M, Faber AC, Wang SE, et al. Acquired resistance to EGFR tyrosine kinase inhibitors in cancer cells is mediated by loss of IGFbinding proteins. J Clin Invest. 2008;118:2609-2619.

56. Franke TF, Hornik CP, Segev L, Shostak GA, Sugimoto C. PI3K/Akt and apoptosis: size matters. Oncogene. 2003;22:8983-8998.

57. Marmor MD, Skaria KB, Yarden Y. Signal transduction and oncogenesis by ErbB/HER receptors. Int J Radiat Oncol Biol Phys. 2004;58: 903-913.

58. Jhawer M, Goel S, Wilson AJ, et al. PIK3CA mutation/PTEN expression status predicts response of colon cancer cells to the epidermal growth factor receptor inhibitor cetuximab. Cancer Res. 2008;68:1953-1961.

59. Bianco R, Shin I, Ritter CA, et al. Loss of PTEN/MMAC1/TEP in EGF receptor-expressing tumor cells counteracts the antitumor action of EGFR tyrosine kinase inhibitors. Oncogene. 2003;22:2812-2822.

60. Engelman JA, Zejnullahu K, Mitsudomi T, et al. MET amplification leads to gefitinib resistance in lung cancer by activating ERBB3 signaling. Science. 2007;316:1039-1043.

61. Ihle NT, Paine-Murrieta G, Berggren MI, et al. The phosphatidylinositol3-kinase inhibitor PX-866 overcomes resistance to the epidermal growth factor receptor inhibitor gefitinib in A-549 human non-small cell lung cancer xenografts. Mol Cancer Ther. 2005;4:1349-1357.

62. Abraham RT, Gibbons JJ. The mammalian target of rapamycin signaling pathway: twists and turns in the road to cancer therapy. Clin Cancer Res. 2007;13:3109-3114.

63. Bjornsti MA, Houghton PJ. The TOR pathway: a target for cancer therapy. Nat Rev Cancer. 2004;4:335-348.

64. Bianco R, Garofalo S, Rosa R, et al. Inhibition of mTOR pathway by everolimus cooperates with EGFR inhibitors in human tumours sensitive and resistant to anti-EGFR drugs. Br J Cancer. 2008;98: 923-930. 
65. Kobayashi S, Boggon TJ, Dayaram T, et al. EGFR mutation and resistance of non-small-cell lung cancer to gefitinib. $N$ Engl J Med. 2005;352:786-792.

66. Kosaka T, Yatabe Y, Endoh H, et al. Analysis of epidermal growth factor receptor gene mutation in patients with non-small cell lung cancer and acquired resistance to gefitinib. Clin Cancer Res. 2006;12: 5764-5769.
67. Bean J, Brennan C, Shih JY, et al. MET amplification occurs with or without T790M mutations in EGFR mutant lung tumors with acquired resistance to gefitinib or erlotinib. Proc Natl Acad Sci USA. 2007;104:20932-20937.

\section{Publish your work in this journal}

Biologics: Targets \& Therapy is an international, peer-reviewed journal focusing on the patho-physiological rationale for and clinical application of Biologic agents in the management of autoimmune diseases, cancers or other pathologies where a molecular target can be identified. This journal is indexed on PubMed Central, CAS,
EMBase, Scopus and the Elsevier Bibliographic databases. The manuscript management system is completely online and includes a very quick and fair peer-review system, which is all easy to use. Visit http://www.dovepress.com/testimonials.php to read real quotes from published authors. 